Report Number COO-1799-8

COALESCENCE OF HELIUM BUBBLES IN ALUMINUM

K. Y. Chen and J. R. Cost

School of Materials Science and

Metallurgical Engineering

Purdue University

Lafayette, Indiana 47907

March 1972

NOTICE

This report was prepared as an account of work sponsored. by the United States Government. Neither the United States nor the United States Atomic Energy Commission, nor any of their employees, nor any of their contractors, subcontractors, or their employees, makes any warranty, express or implied, or assumes any legal liability or responsibility for the accuracy, completeness or usefulness of any information, apparatus, product or process disclosed, or represents that its use would not infringe privately owned rights. 


\section{DISCLAIMER}

This report was prepared as an account of work sponsored by an agency of the United States Government. Neither the United States Government nor any agency Thereof, nor any of their employees, makes any warranty, express or implied, or assumes any legal liability or responsibility for the accuracy, completeness, or usefulness of any information, apparatus, product, or process disclosed, or represents that its use would not infringe privately owned rights. Reference herein to any specific commercial product, process, or service by trade name, trademark, manufacturer, or otherwise does not necessarily constitute or imply its endorsement, recommendation, or favoring by the United States Government or any agency thereof. The views and opinions of authors expressed herein do not necessarily state or reflect those of the United States Government or any agency thereof. 


\section{DISCLAIMER}

Portions of this document may be illegible in electronic image products. Images are produced from the best available original document. 


\section{ABSTRACT}

The formation and coalescence of helium bubbles in aluminum with annealing at $550^{\circ} \mathrm{C}$ is examined experimentally using electrical resistivity and electron microscopy. Results are compared with those obtalned by computer simulation in order to test models based upon various mechanisms for bubble motion. The computer method previously developed is extended from an ideal to a Van der Waals gas and both upper and lower limiting values of the surface energy of aluminum are considered. These factors are shown to significantly affect the kinetics of coalescence. Comparison of experimental and computer results indicates that the motion of helium bubbles in aluminum is not surface diffusion limited and that it is consistent with the facet nucleation-limited mechanism originally proposed by Willertz and Shewmon. 


\title{
COALESCENCE OF HELIUM BUBBLES IN ALUMINUM
}

\author{
K. Y. Chen and J.R. Cost*
}

\section{INTRODUCTION}

It has long been recognized that the main energy resource in the near future may be nuclear energy. One of the problems encountered in nuclear reactor design is fuel swelling. Swelling is, at least in part; due to inert gases which are neutron-transmutation products. At most operating temperatures, the inert gas atoms precipitate to form gas bubbles which migrate and coalesce to create a greater specific volume per gas atom. To minimize swelling in the optimum design of the fuel or fuel casing materials, the latter process, coalescence, in particular, needs to be further understood. The coalescence of bubbles can be expected to depend in a complicated way upon the size distribution of bubbles, the deviation of the gas in the bubble from ideal gas behavior and other factors. The kinetics of the process, however, depend upon the rate at which bubbles diffuse through the solid, and thus the process of bubble diffusion itself requires careful study.

The early diffusion theories ${ }^{1-3}$ predicted that if surface diffusion were the rate-controlling step in the motion of bubbles, the bubble diffusion coefficient should vary as inverse radius to the 4 th power and should have the same activation energy as the process of surface self-diffusion. Recent experiments on diffusion of bubbles in uranium dloxide ${ }^{4}$, gold and copper ${ }^{5}$, and a stainless steel, ${ }^{6}$ however, showed that surface diffusion alone cannot

\footnotetext{
* School of Materials Science and Metallurgical Engineering, Purdue University, Lafayette, Indiana 47907.
} 
explain the motion of bubbles in these metals. An alternate hypothesis, that the nucleation of facets on the bubble surface was rate-limiting, was recently offered by Willertz and Shewmon ${ }^{5}$. They measured the rate at which individual helium bubbles moved at varlous temperaures and compared these rates with theoretical predictions based upon the two models. Satisfactory agreement was found with the facet nucleation rate-limiting model, but precise quantitative agreement was not possible because the model depended sensitively upon the value of the energy per unit length of the edge (step) of a terrace which exists on the facet, and this value was not known with sufficient accuracy.

It is the purpose of this study to further test and examine the facet nucleation model by comparing predicted and experimental results for the kinetics of bubble coalescence. Here the kinetic behavior of the overall bubble population is to be considered. A computer method to obtain the size distribtuion of bubbles as a function of time has been developed by Gruber $^{2}$. He employed the ideal gas law and a bimolecular reaction equation to evaluate the concentration of various species at various annealing times. The calculation was made possible because there was no dissociation reaction. In the present study, the computer methods of Gruber have been extended to predict the bubble coalescence kinetics for a real, as opposed to an ideal gas, and for several models of facet nucleation-limited, as opposed to surface diffusion-limited, bubble migration. 
EXPERIMENTAL METHOD

All measurements were made on a starting material of high purity aluminum (Cominco American Incorporated, six 9's) which was reduced in thickness to 125 microns by rolling and then annealed at $550^{\circ} \mathrm{C}$ for one hour followed by furnace cooling to $200^{\circ} \mathrm{C}$ and air cooling to room temperature. This treatment resulted in a low dislocation density and minimal dislocation substructure as determined by electron microscopy. After annealing, helium was infected into specimens according to the following procedure.

\section{Irradiation}

Multiple specimens in the form of a stack of five folls, each roughly $1.27 \mathrm{~cm} \times 3.8 \mathrm{~cm} \times 0.0125 \mathrm{~cm}$, were cyclotron irradiated to roughly 50 at. ppm with $46 \mathrm{MeV}$ alpha particles. Uniformity of the helium atoms along the depth of foils was achieved by using a rotating variable-thickness disc of aluminum to degrade the energy. During irradiation, specimens were cooled by circulated water. Previous measurements indicated that using this specimen configuration and cooling method, the temperature of the specimens during irradiation was below $100^{\circ} \mathrm{C}$; however, it is difficult to measure the temperature of each of the folls, and those furthest from the water may have been above this temperature. After irradiation, because of high radioactivity, which was believed to be produced by impurities in the cooling water, the folls were stored at room temperature for elght days. Subsequently, specimens for resistivity measurement and electron microscopy were prepared. Some differences undoubtedly exist in the character and the degree of the radiation damage which occurred to the different folls in the *Argonne National Laboratories, Argonne, Ill. 
stack. This is because the number of incident particles passing through any particular region of foil was, in this irradiation, decreases with the distance from the incident surface. It may be assumed, however, based upon evidence that irradiation-induced point defects anneal out below $100^{\circ} \mathrm{C}$ in aluninum ${ }^{8}$, that these differences are not appreciable.

2. Resistivity Measurement

For resistivity measurements, specimens, approximately $3 \mathrm{~cm}$ long and. $0.15 \mathrm{~cm}$ wide were cut from the folls by electropolishing. For measuring the potential drop, $0.0125 \mathrm{~cm}$ diameter leads of high purity aluminum wire were then attached by spot welding. A helium-injected specimen was connected in series with a non-irradiated specimen so that during the resistance measurement the same current passed through both. All measurements were made in liquid helium at a current of $1 / 2$ ampere suppiled by a constant current source. The potential drop across the specimen was measured with a sensitivity of roughly one nanovolt using a six-dial potentiometer in conjunction with a photocell galvanometer-amplifler. Because the resistivity effects were known to be small, extreme care was taken to reduce and correct for thermal emfs. The length to area ratio of the specimen was measured as 1670 per $\mathrm{cm}$, and the sensitivity of the system for resistivity was roughly $10^{-12} \mathrm{ohm} \mathrm{cm}$. The accuracy, however, was limited to one order of magnitude lower, mainly because of variations in the thermal emfs in the leads. These varlations are believed to be due to changes in junction characteristics with annealing at high temperature $\left(550^{\circ} \mathrm{C}\right)$. The resistivity ratio for a 115 micron thick specimen was $R_{298^{\circ}} / R_{4.2^{\circ} \mathrm{K}}=3180$. To prevent oxidation of the potential leads during the high temperature anneals, the electrical resistivity specimens were annealed in a helium atmosphere. Also the specimens were held at $180^{\circ} \mathrm{C}$ for 5 minutes during cooling from this anneal in 
order to allow the supersaturated vacancy concentration to anneal out.

\section{Electron Microscopy}

Because we are interested in bubbles in a size range near the lower resolution limit of the microscope, extreme care was exercised in the electropolishing and thinning techniques used to prepare specimens. Discs, $2.3 \mathrm{~mm}$ in diameter, were punched out from the foil and further thinned by mechanically polishing on both surfaces. It was found that removal of the surface layer by mechanical polishing was necessary to consistently obtain high quality specimens. In the next step, specimens were thinned to perforation with a twin jet electropolisher in an electrolytic solution of one part perchloric acid and 4 parts ethanol which was kept below $-10^{\circ} \mathrm{C}$ during the polishing. Specimens were then examined by transmission electron microscopy at $100 \mathrm{KV}$. Because of difficulty in obtaining thin areas of suitable orientation, two discs were often made for each annealing specimen. The use of mechanical polishing added some complications. First, it became a problem to obtain specimens of reasonable flatness as evidenced by the abundance of bend extinction contours, and second, it became difficult to find dislocationfree regions. This latter problem was considered important because of the possible effect which the presence of dislocations might have upon the kinetics of bubble coarsening. Such an effect could be due to dislocation pinning of individual helium atoms and/or gas bubbles. In choosing representative regions, we were usually able to avoid such areas.

To obtain optimum contrast, a low order beam was used ${ }^{9}$ as much as possible. The exception to this was when the foil had no area in a suitable orlentation. Quantitative measurements of bubbles were always made at 
In-focus imaging conditions and at the exact Bragg condition $9-11$. The thickness determination of a given area was made from slip traces or thickness contours at the same diffraction condition $(s=0)$. The error involved in this determination is estimated to be roughiy $25 \%$. For bubble radil smaller than $\sim 15 \mathrm{~A}$, the bubbles are visible only in the out-of-focus condition and the real size may vary considerably from that measured in the micrographs ${ }^{11}$. Hence the size range of our main interest is above the $15 \mathrm{~A}$ radius limit. Bubble diameters were measured with a filar eyepiece on micrographs enlarged at either $1.3 \times 10^{5}$ or $2 \times 10^{5}$. The size of the bubble was taken as the diameter of the center of the annular ring of highest contrast which appeared around the bubble. Examinations of more than one area were usually made to obtaln the bubble size distribution.

4. Computer Calculation

In order to compare experimental results with various mechanisms for bubble coalescence, we employed the method using a digital computer first adopted by Gruber ${ }^{2}$. His method is based upon the bimolecular reaction equation,

$$
\Delta F_{i j}=4 \pi D_{i j} r_{i j} F_{i} F_{j}\left\{1+\left[r_{i j} /\left(\pi D_{i j} t\right)^{\frac{1}{2}}\right]\right\} \Delta t_{g}
$$

given by Chandrasekhar ${ }^{12}$, where $\Delta F_{i j}$ is the number of collisions between $\mathcal{I}$ and $j$ bubbles in the time interval $\Delta t, D_{i j}$ is the sum of the diffusivity of the $i$ and $f$ bubbles, $r_{i j}$ is the sum of their radil, $F_{i}$ and $F_{j}$ are their respective concentrations, $t$ is the elapsed annealing time and $i$ and $j$ denote the number of atoms in the given bubbles. In considering the two terms in the brackets in this equation, the first term can be viewed as 
determining the number of events in time $\Delta t$ which take place after the bubble concentrations have developed non-random distributions. These nonrandom distributions are set up because two colliding bubbles act as sinks for each of their species and thus create concentration gradients. Before these concentration gradients are set up, there is then an initial transient In the annealing rate due to the fact that the distribution is initially random. This transient, which is removed early in the annealing, is covered by the second term in the brackets. It was found early in our study by systematic comparison of results, that, for our helium concentration, bubble size range, and annealing time of interest, the second term was not a significant correction. Thus the results reported have for the most part been obtained by using only the first term, as was done by both Chandrasekhar and Gruber.

The method used with the Chandrasekhar equation was to calculate all! the possible collisions between bubbles of all sizes (species) for a given time interval. The resulting concentration of each new species bubble was then stored in the computer memory before the reactions for the next step were calculated. Gruber's original calculations employed the ideal gas equation; this had the advantage of simplifying both the analysis and the results. Since we are dealing with a real gas in this study, the Van der Waals equation with the valid constant must be used,

$$
p(v-m B)=m R T \text { : }
$$

Here $p, v, T, R$, and $m$ are pressure, volume, temperature, the gas constant and the number of moles respectively, and $B$ is the Van der Waals constant. The correction from an ideal to a Van der Waals gas somewhat complicates the calculation and the analysis of the kinetics of coalescence. It is, 
however, a significant correction, especially early in the process when the bubbles are small and are at high pressure.

Several parameters associated with the coalescence calculation should be mentioned. These are (1) the number of different specles to be kept in the memory, (11) the conversion of species to a different size range in order to allow conslderation of the newly created larger species, and (1ii) the selection of the time interval for each increment of the calculation. Effects of varying each of these parameters were studied, and the final choices were made to keep the total error small while keeping computer time within reason. For the number of species in the memory, it was found that the difference in results between 20 and 100 species could be ignored; most of the calculations were made using 20 or 30 species in the memory. For the second parameter, the conversion of species to a different size range, the size range was doubled when the ratio of the concentration of ! the largest species to that of the most populated one exceeded $0.01 \%$. It was found that this procedure resulted in errors of $1.3 \%$ and $2.2 \%$ in the mean bubble radius and the bubble density respectively during a single conversion. Calculations starting with single helium atoms usually required ten to eleven conversions, while calculations which started with small observable bubbles required only one conversion. These latter calculations were the ones compared with our experimental coalescence results. Variation of the third parameter, the time interval, $\Delta t$, was found not to produce significant changes except for very early annealing times where the elapsed time is of the order of $\Delta t$. A value of $\Delta t$ chosen on the basis of a $5 \%$ decrease in the population of the most populated species was used and was quite satisfactory. 


\section{EXPERIMENTAL RESULTS}

\section{Electrical Resistivity}

The electrical resistance at $4^{\circ} \mathrm{K}$ of helium-injected specimens was found to decrease rapidly with annealing at $550^{\circ} \mathrm{C}$, while that of pure aluminum dummy specimens did not. In Fig. I the difference in resistivity between the two specimens is plotted and may be seen to decrease monotonically during the first hundred minutes of annealing. The initial difference in resistivity, $5.3 \times 10^{-9} \mathrm{ohm} \mathrm{cm}$, can be interpreted as the resistivity contribution due to injected helium. The final constant difference in the resistivities of the two specimens, roughly 2 micro-ohm $\mathrm{cm}$, appears to be due to the presence of helium in the form of bubbles.

\section{Electron Microscopy}

Typical results obtained by electron microscopy of specimens annealed to cause precipitation and growth of helium bubbles are shown in Fig. 2. Here the specimen was annealed at $550^{\circ} \mathrm{C}$ for $6.12 \times 10^{4} \mathrm{sec}$, an anneal which results in the full, decrease in the electrical resistivity. The bubbles have radii in the size range from 25 to $65 \mathrm{~A}$. Also in Fig. 2, we note that the bubbles appear to have faceted surfaces: Faceting of bubbles was resolved when the bubbles were of appropriate size and imaging conditions were suitable. Faceting in aluminum has been previously reported ${ }^{13}$ and its presence is significant if the facet nucleation-limited coalescence hypothesis is to be tested.

The contrast of bubbles in the electron microscope varies with imaging conditions. As has been shown 9,10 by both two beam calculation and experimental observation, at an exact Bragg condition $(s=0)$, bubble contrast 
reverses across a thickness contour. A similar example of the phenomenon of contrast reversal for a bubble is shown in Fig. 3 where reversal is observed across a bend extinction contour with three (including two [1II]) beams operating.

Comparison of Fig. 3. with Fig. 2 demonstrates the phenomenon of coalescence. The specimen shown in Fig. 3 was annealed at $550^{\circ} \mathrm{C}$ roughly ten times longer than that in Fig. 2. As can be observed in the figure, this resulted in larger bubbles for the longer annealing time. Not apparent, but measured by examination of larger areas of specimen, is a significant decrease in the bubble density with annealing.

The kinetics of the process of coalescence has been studied by examining the bubble size distribution as a function of annealing time at a constant temperature. The results of such an analysis of specimens annealed at $550^{\circ} \mathrm{C}$ are shown in Table I. These data were obtained by classifying according to size the total observable bubble population in one or more areas of specimens annealed for various times. It is considered that the number of uncounted bubbles was not significant first, because we used the exact Bragg condition $(s=0)$ with a low order beam, second, because the observed frequency distributions tail off above the lower resolution limit of the microscope, and third because, for calculations based upon the expected surface energy of aluminum, all the hellum injected was accounted for in the bubbles observed. (This latter point will be discussed in greater detail.) For several reasons, the number of bubbles in each of the populations presented in Table $I$ is small for usual purposes of sampling statistics. For highly accurate characterization of the size distributions, considerably more data are required. These data are, however, not easy to 
obtain because of the Iimited amount of irradiated specimen and the limited amount of thin, flat area of suitable orlentation on each microscopy specimen. Also the exclusion of non-representative areas, such as those with a high dislocation density, where traps for helium atoms or bubbles existed during annealing, limited the size of the population which could be studied. Fortunately, the changes in the size range and the magnitude of the bubble distribution were relatively large so that, although the accuracy was not high, significant changes could be observed. This may be seen in Fig. 4, where the bubble distributions for the first and the fourth annealing times presented in Table I, are plotted. It may be noted that these data are plotted in terms of $n$, the number of gas atoms per bubble. This is the unit of bubble size used in Gruber's and in our computer calculations. The proper conversion formula will be shown in the next section.

Gruber also presented a theoretical analysis of the shape of the size distribution during isothermal annealing. The generalized result he obtained has been fitted to our data, as shown by the curves in Fig. 4 . Both the experimental and the theoretical size distributions show an increase in the mean number of atoms per bubble and a decrease in the total number of bubbles with annealing. It will be shown later that the distributions obtained using the ideal gas law are not appreciably different from those using the Van der Waals equation, thus agreement between the experimental and the computer calculated distributions is expected. The results of using Gruber's fit to our data indicate that $D_{s}$ the surface diffusion coefficient is of the order $10^{-11}$ to $10^{-12} \mathrm{~cm}^{2} / \mathrm{sec}$. Based upon best estimates of $D_{s}$ to be discussed later, this is roughly six orders of magnitude too small, and suggests that in this study, bubble migration is not limited by surface 


\section{diffusion alone.}

\section{Computer Calculation}

In Figs. 5 and 6 are shown log-log plots of the computer calculated bubble density and mean bubble radius respectively as a function of annealing time. The equations shown on Fig. 5 are those for the bubble diffusivities which were used in Eq. I to calculate the annealing curves. The equation for the surface diffusion-limited bubble motion is due to Gruber, and that for facet nucleation-limited motion is based upon a modification of the model proposed by Willertz and Shewmon ${ }^{5}$, as is described in the Appendix. For comparison with our experimental results, all calculations have been made for an initial concentration of helium atoms of $3 \times 10^{18}$ per $\mathrm{cm}^{3}$ (50 at. ppm), and an annealing temperature of $550^{\circ} \mathrm{C}$. Also $\mathrm{D}_{\mathrm{s}}$, the surface diffusion.coefficient is assumed to be $10^{-5} \mathrm{~cm}^{2} / \mathrm{sec}$ and $r$, the surface energy, is taken to be 150 ergs per $\mathrm{cm}^{2}$. Since data on the surface self-diffusion on aluminum are not in the literature, we use a typical value of $10^{-5} \mathrm{~cm}^{2} / \mathrm{sec}$, estimated at the same fraction of the melting point from the available data for the fcc metals $\mathrm{Au}^{14}, \mathrm{Cu}^{15}$ and $\mathrm{Ag}^{16}$. Discussion of the value used for the surface energy will be given later in this paper.

The results for an ideal gas agree with those of Gruber and predict that after the short initial transient required to form an average bubble radius of $3 A$, the bubble density decreases as $t^{-2 / 5}$ and the average bubble radius increases as $t^{1 / 5}$. For the Van der Waals gas with the proper constant for hellum, the coalescence process is predicted to be somewhat delayed compared to the ideal gas, see Fig. 5. After the initial delay, the rate then becomes greater than that for the ideal gas, and the kinetics of the two 
processes become almost identical late in the anneal, where the Van der Waals correction is less important because the pressure in the bubbles is much less. Neither of these predicted annealing curves provide good agreement with our experimental results. For instance, they both predict that an average bubble radius of $50 \mathrm{~A}$ will be reached in less than $1 \mathrm{sec}$, while experimentally this average size was never obtained, even after annealing for $6.55 \times 10^{5} \mathrm{sec}(182 \mathrm{hrs})$.

The computer calculated results for coalescence which is facet nucleation-limited are shown by the right hand curves in Figs. 5 and 6 . The model for facet nucleation is one of two such models discussed in detail in the Appendix. It is based upon the assumption that motion of a bubble depends upon the nucleation of a new layer of atoms on one of the facets of the bubble, and that the critical nucleus is a layer, the edge of which extends across the facet at its "diameter". It may be noted that the facet nucleation models predict a variable time exponent as opposed to the constant exponent for the surface diffusion-limited models. Also facet nucleation shows better agreement with experiment. In comparing the computer results of the different models, it also is of interest to consider the shape of the bubble size distribution at the same average bubble radius. The shape was found to be essentially the same for the surface diffusion mechanism as for that of facet nucleation, independent of whether the gas was ideal or a Van der Waals gas.

The two models described in the Appendix were chosen since they represent two extremes in the energy of the critical nucleus for the for-. mation of a new layer on a facet. As previously mentioned in discussing . 
Flg. 5, the first model is for a critical nucleus which has an edge extending across the facet diameter. In the second model, the emitting and filling of two different facets on a given bubble are considered, and the condition for a critical nucleus is determined by the energy maximum for the whole system. The equations for both of these models are similar in that they both have a dominant factor which gives an exponential dependence of the bubble diffusivity upon the bubble radius. The important parameter weighting this exponential dependence upon radius is $\epsilon$, the energy per unit length of the step of a new layer which nucleates on a facet. The effect of varying this edge energy for the two extreme models considered, is shown in Fig. 7 , in which the variation of the mean bubble radius with annealing time is plotted. Also plotted is the mean bubble radius obtained from the electron microscopy results listed in Table $I$. The initial distribution used in the computer calculation was taken from the data obtained at $550^{\circ} \mathrm{C}$ for $6.1 \times 10^{4} \mathrm{sec}$. One notes that there is considerable scatter, roughly $10 \%$, in the experimental values for the average radius. This appears to be due to the difficulties in sampling as previously discussed. It should also be noted that to fit the data in. Fig. 7 to a surface diffusion limited bubble motion, requires a surface diffusion coefficient approximately six orders of magnitude smaller. This again suggests that surface diffusion is not the rate-limiting mechanism in bubble motion, the same conclusion we derived from analysis of the bubble size distributions in Fig. 4. 


\section{DISCUSSION}

\section{The Van der Waals Constant}

From the Van der Waals equation and the relation $p=2 \gamma / r$ for the pressure of a bubble in equilibrium with the surrounding matrix, the number of moles of helium in a bubble is given by

$$
m=\frac{8 \pi \gamma r^{2} / 3}{R T+2 \gamma B / r}
$$

Thus the properties of a system which determine the number of gas atoms per bubble are the surface energy of the matrix material and the Van der Waals constant of the gas.

In the course of this study, the proper Van der Waals constant for annealing of helium bubbles was considered, and a-different value than has been previously used is recommended. The Van der Waals constant $B=24 \mathrm{ml} /$ mole, quoted ${ }^{17,18}$ most often for helium appears to be derived from data obtalned at low pressure and low temperature. For annealing studies of helium in metals, this constant should be corrected to a high pressure and high temperature value: Bridgman ${ }^{19}$ studied helium gas at high pressure, up to roughly $1.5 \times 10^{4}$ atmospheres $\left(\sim 1.5 \times 10^{10}\right.$ dynes $\left./ \mathrm{cm}^{2}\right)$, and Schneider and Duffie 20 obtained the constant B for various temperatures at pressures up to roughly 75 atmospheres $\left(\sim 7.6 \times 10^{7}\right.$ dynes $\left./ \mathrm{cm}^{2}\right)$. Because experimental results obtained simultaneously at high temperature and high pressure have not been reported, we have examined both of these sets of data. An interpolation of of Schneider and Duffie's data yields $B=9.98 \mathrm{ml} / \mathrm{mole}$. When this corrected value of $B$ is used to obtain the number of gas atoms per bubble, a larger number is obtained compared to previous values, see the curves for $\gamma=$ $150 \mathrm{ergs} / \mathrm{cm}^{2}$ in Fig. 8. The difference is significant and increases for 
the smaller bubbles in which the pressure is higher. This difference also increases with an increase in surface energy assuming a given bubble size.

In the high pressure regime in Bridgman's data, the empirical relation $\mathrm{pV}=1.4 \mathrm{RT}+\mathrm{B}_{\mathrm{T}} \mathrm{p}, \mathrm{B}_{\mathrm{T}}=9 \mathrm{ml} / \mathrm{mole}$ is obtained. The result employing this equation to give the number of gas atoms per bubble as a function of the radius, see Fig. 8, is in good agreement with that obtained by using the Schneider and Duffle value of $B$ in the Van der Waals equation, particularly for high pressure (1.e., for small bubbles). Certainly the differences are small compared with those obtained by using the previous value of, B. On this basis and for simplicity in our discussions, we use Schnelder and Duffie's value, $B=9.98 \mathrm{ml} / \mathrm{mole}$, for our calculations. It may also be pointed out that consideration of the effects of temperature and pressure in selecting the Van der Waals constant becomes particularly important for inert gases other than helium which have larger deviations from ideal gas behavior. 2. The Surface Energy of Solid Aluminum in Helium

Eq. (3) can be used to obtain the number of moles of helium in the bubbles in the various distributions examined. Alternately we have calculated $\gamma$ using Eq. (3) by requiring that the total moles in foils for which the thickness has been measured agree with the injected concentration of helium. The results of this analysis, partially because of the relatively large uncertainty in the measurement of film thickness, and therefore bubble density, indicate $\gamma$ to be in a range from 150 to $1000 \mathrm{ergs} / \mathrm{cm}^{2}$. Although measurements of the surface energy of solid aluminum do not appear in the literature, the latter value is in agreement with the rough value of 900 ergs $/ \mathrm{cm}^{2}$ for liquid aluminum near its melting point ${ }^{21}$. 
Values of $\gamma$, at the extremes of the range indicated, are the values which have been used in Fig. 8 .

Because of the extremely high pressures of helium, up to roughly $10^{4}$ atmospheres $\left(\sim 10^{10}\right.$ dynes $\left./ \mathrm{cm}^{2}\right)$, which are present in the small bubbles, it is possible that the surface energy of aluminum in equilibrium with this gas may be decreased from the value at zero pressure. Such a decrease might be expected on the basis of the increased interaction which a gas at high pressure will have with the surface. Certainly this is true when a gas near its triple polnt is liquified by high pressure. Since the value of $\gamma$ is only known within the range limits 150 to $1000 \mathrm{ergs} / \mathrm{cm}^{2}$, we have made the computer calculations of coalescence using both the limiting values for $\gamma$. Usually the resulting annealing curves have been relatively insensitive to the value chosen. On the basis of the effects of extremely high pressure upon $\gamma$, we have presented the computer results using the lower value of $\gamma, 150 \mathrm{ergs} / \mathrm{cm}^{2}$.

\section{Initial Formation of Bubbles}

Several points can be made comparing the actual kinetics of precipitation of bubbles, as deduced from the resisitivity results, with the predicted results based upon the computer calculation. Previous resistivity and lattice parameter data for isochronally annealed specimens heated for $30 \mathrm{~min}$ at $25^{\circ} \mathrm{C}$ temperature intervals indicate that bubble formation is nearly complete by the time the specimen is heated to $550^{\circ} \mathrm{C}$. The results of the present study are in full agreement with those of the earlier one, and in addition they indicate that atomic helium is no longer present in the lattice after only $100 \mathrm{~min}$ at $550^{\circ} \mathrm{C}$. For such short annealing times, the presence of bubbles could not be determined with certainty using the electron microscope. However, it is considered that 
they had already formed since, if the helium were present in another form, such as in some type of cluster, presumably the resistivity would show a further decrease when bubbles finally precipitated.

The lack of change in the resistivity between 100 and 1000 minutes is significant since bubble coalescence is occurring during this time, as Indicated in Fig. 7 by the steep increase of the mean bubble radius with time at 1000 minutes. This finding that coalescence produces no appreciable change in the resistivity has physical basis. Roughly, the amount of extra scattering of the conduction electrons by the bubble should be proportional to the surface area of the bubbles. Similarly, for the rough assumption of an ideal gas, the total number of moles of helium, which is constant, is proportional to square of the radius, Eq. (3) with $B=0$. Thus the extra scattering may be considered constant independent of changes in the bubble size distribution. (This should also be roughly true for a non-ideal gas as long as the moles of helium per bubble is proportional to the radius to some power which is roughly constant. This is true for the size range studied since in Fig. 8, the slope changes negligibly in the radius range from 20 to $50 \mathrm{A.})$

The precipitation of bubbles was also simulated by computer calculations. Certainly in the regime in which bubbles are smaller than the size of a critical nucleus, the Gruber method is inapplicable. One does not expect a cluster of two or three helium atoms to behave as a bubble. In addition, assuming that the critical nucleus has at least three helium atoms, it is expected that sub-critical nuclei will dissociate, and this reaction was not considered in the computer calculations. In essence, then, the computer calculations do not consider' the time for nucleation, and thus 
they will tend to predict faster coalescence. This will be true even though the proper model and the correct parameters have been used. Moreover, the bubble diffusion coefficient used in the calculation may not be applicable for small bubbles for which a facet cannot be well defined. These discrepancies can, however, be adjusted by ignoring the actual starting time for annealing and considering that the process actually starts when bubbles are just larger than the critical nucleus size. To be complete, this adjustment requires a correction for the bubble concentration, since it is changing as nucleation occurs. The easy solution to this is to consider that there is some "effective starting time" at which the bubbles are all nucleated and at which coalescence starts. Using these ideas, then the computer generated curves should have the portions eliminated which have radii smaller than the radius of the critical nucleus. Also the curves should be translated along the time scale so that the critical size nucleus exists at the "effective starting time." The computer calculated curves and the experimental data can then be compared. Such a comparison has been made in Fig. 7 .

4. Models for Facet Nucleation

It is worthwhile to consider in some detail alternatives to the willertz and Shewmon model for facet nucleation. Models may well vary in the position and shape of the critical nucleus as well as take into consideration the total edge energy of all of the facets on a bubble. In the remainder of this section, two models which represent extremes in the critical nucleus concept (and in the activation energy for bubble diffusion) are considered in detail. Expressions for the bubble diffusivity for these models are formulated in the Appendix. 
Both of the models to be discussed consider a helium bubble to be a polyhedra with many sides or facets. A study of the shape of pores in aluminum at thermal equilibrium ${ }^{13}$, indicates that aluminum is faceted on the $\{100\},\{110\}$ and $\{111\}$ planes so that twenty-six facets make up the bubble. Also, the energles of these planes are equal within a few percent so that the different facets have nearly equal area.

\section{(a) Emitting Ledge Model}

This model considers that nucleation occurs in a relative simple fashion. Atoms either arrive at or leave a facet which is initially atomically smooth. Arriving atoms which stay on the facet will tend to form: a new layer starting at the edges of the facet. Thus the edges of the empty facet act as a ledge which is always present and which arriving atoms can attach to. Because of this, an isolated new layer in the center of the facet (referred to as a pillbox by Willertz and Shewmon) will not tend to form. As more atoms arrive and attach to edges of the new layer, the new layer will tend to develop a shape which minimizes the total edge length. Because of its large amount of edge length per unit area of layer deposited, a circular shaped ledge (with the new layer outside of the circle) is unstable with respect to a shape which has the edge of the new layer as a chord between edges of the facet. Such a sub-critical nucleus, with an edge which is a chord may be expected to form either by fluctuations in the deposition rate to various portions of the facet or by the continuous rearrangement of the atoms on the ledge. The layer with a chord edge can then grow across the facet to become a critical nucleus which is a layer having an edge that is a facet diameter. Such a critical nucleus has the least edge length, of all the models we considered, and thus it is expected to be the most probable. Whether it occurs frequently depends upon whether it is able to.form, and this would depend upon whether the initial edge shape which is presumably a polygon formed 
by attaching atoms to the edges of the facet, can convert into a chord across adjacent edges of the facet.

Atoms must be emitted from another facet for the above process to take place. Emission from a facet can also result in an extra layer with a straight edge which is a chord across the facet. For an empty facet, emission can be expected to start with a surface vacancy which will tend to form with equal probabllity anywhere on the surface of the facet, except at the edges. Emission of the adjacent atoms to the surface vacancy will then develop a layer of missing atoms. This layer will tend to be circular to minimize edge length. Such a structure has been described by Bethge $^{22}$ as a disc-shaped hole one atom in depth and was given the name "Lochkeime," or embryo cavity. We will use the nomenclature of Willertz and Shewmon who use the term "pillbox" for a new layer which forms above an existing layer of the facet. Thus, the structure we are dealing with here is a "negative pillbox". Growth of this empty layer by further emission will ultimately result in impingement with the facet edges. For the special case in which the initial surface vacancy was near the center of the facet, this impingement will result in a critical nucleus since the sharp corners of the edge, which occur at the point of impingement will tend to straighten as further emission occurs, and this will result in a decrease in the edge length as the area of the layer increases. For the more frequent case where nucleation is not near the center of the facet, impingement will result in the negative pillbox forming into a partial layer (negative) with an edge which is a chord between facet edges. Again, such a changè in shape will tend to occur 
because it decreases the edge length. It is expected to be able to occur because of the relatively high mobility of atoms at the edge of a layer. The formation of the critical nucleus for a new layer from this partial layer with a straight edge is then the same as for the filling ledge; the layer grows by emission until the chord becomes a diameter. Although the critical nuclei are the same for the two processes, it is considered that emission from a facet is more difficult than filling. This is because of the differences in nucleating the initial new layer. For emission this requires a surface vacancy to be formed, while for filling, pre-existing nuclei are present in the form of the facet edges. This reasoning leads one to conclude that the rate-limiting step in formation of new facets is the creation on an emitting facet of a ledge which is a facet diameter. (b) Two-Pillbox Model

During bubble diffusion, the total step energy in both the emitting and filling facets undergoes a maximum. If atoms are emitted from near the center of a facet and migrate to and deposit preferentially at the edge of another, both emitting and filling facets become negative pillboxes. The size of the critical nucleus is determined by the maximum in the edge energy of the system composed of all the facets and their pillboxes. An equation for the bubble diffusivity when the exchange of atoms is between just two facets is developed in the Appendix.

A facet with no ledge or pillbox on it is in the lowest energy state. It can be shown using even modest values of the ledge energy that the fraction of the time that a facet will have a ledge on it should be very small. It is thus reasonable to consider the case where both facets are initially without ledges. Also, because of the small area of a facet of our interest, and the relatively high energy associated with surface defects of atomic size, 
the concentration of surface vacancies and adsorbed atoms is considered to be negligibly small.

For two initially empty facets, the formation of a critical nucleus requires emission of atoms from the center of one facet forming a negative pillbox which is growing outward, and filling of atoms starting at the edges so that the second facet also has a negative pillbox, but one which is growing inward. The total energy of the system goes through a maximum when both pillboxes are the same size. Thus critical nuclei are created on each facet at the same time. This is obviously a very special case. The more general case in which initially one of the facets is partially filled, is interesting to consider, but more complicated. Also, one can show that if one of the facets is initially partially filled, then the energy for the critical nucleus is increased. This suggests that bubble motion will then change markedly due to the presence of a few extra atoms on the bubble surface. Certainly such a complicated dependence of diffusivity upon bubble size would be difficult to establish.

5. Migration and Coalescence of Helium Bubbles

Since bubble growth in inert gas-metal systems is believed to occur by a mechanism of bubble migration, collision, and coalescence, and since this growth is, in general, undesirable, then any means by which bubble motion might be retarded will be of great engineering interest. Thus the finding that the motion of bubbles in aluminum is slower by several orders of magnitude than that predicted by a surface diffusion-limited mechanism requires more careful examination.

The predicted kinetics of coalescence for the facet nucleation model is, as shown in Figs. 6 and 7, a very rapid growth, once the bubbles them- 
selves have nucleated, followed by a sharp decrease in the growth rate until it becomes almost negligible. (At $10^{6} \mathrm{sec}$ in Fig. 6 the growth rate is proportional to $t^{0.05}$.) The result of this is an effective "terminal bubble size." An example of the terminal size may be obtained by examination of Fig. 7. Here it is shown that experimentally bubbles nucleate and grow to an average radius of $40 \mathrm{~A}$ in roughly $2 \times 10^{5}$ seconds, but after $6 \times 10^{5}$ seconds they have only grown to $45 \mathrm{~A}$. Also calculation (from Fig. 6) of the radius after annealing for one year $\left(\sim 3 \times 10^{7} \mathrm{sec}\right)$ gives only $50 \mathrm{~A}$. This result is in contrast to that predicted by the surface diffusion model which predicts that the bubble size increases continuously according to $t^{0.2}$. The key finding is that the experimental results qualitatively agree with the predictions of the facet nucleation model. Of course, the reason that such a model leads to a terminal bubble size is because it predicts that the bubble diffusivity depends exponentially upon the bubble radius, see equations in Fig. 7. The pre-exponential radius terms have little effect; when the bubble reaches a certain size 1t, for all practical purposes, stops moving.

The factor other than the radius wich determines the terminal bubble size is the edge energy per unit length, $\epsilon$. The edge energy is expected to be constant for a given material, but unfortunately, it is only known for a few materials. Thus, in order to fit our data in Fig. 7, we have used $\epsilon$ as an adjustable parameter in each of the equations. It may be seen that the large values for the edge energy result in smaller terminal bubble sizes. Also the coalescence kinetics predicted by the two models are almost indentical when the multiplying factors for the radius in the exponential term ( $0.8 \epsilon$ and $3.55 \epsilon$ for the two models) is the same. Typical 
values of $\epsilon$ which have been reported 5,23 , indicate that it is very roughly in the range from $2.5 \times 10^{-6}$ to $4.0 \times 10^{-6}$ ergs per cm. In Fig. 7, it may be seen that the values of $\epsilon$ are $9 \times 10^{-6}$ and $2 \times 10^{-6}$ ergs per cm for the emitting ledge and the two-pillbox models respectively. This suggests that neither of the models considered is correct and that the proper one will be intermediate between the two, but perhaps closer to the more complicated two-pillbox model.

If one closely considers the shape of the experimental and computer calculated curves in Fig. 7, it may be observed that none of the curves which were calculated in an attempt to fit the data are able to provide a good fit. The scatter in the data prevents statement of a firm conclusion, but since neither of these equations adequately describe the kinetics in this system, then, if coalescence is indeed facet nucleation-limited, presumably the process is more complex than has been described by the theories and models considered to date. 
APPENDIX

Here expressions for the bubble diffusivity will be developed, following the method of Willertz and Shewmon, for the two models for nucleation on facets which were previously described. For mathematical convenience, although the facets are actually polygons with a number of sides determined by the symmetry of the particular surface, they will be approximated by circles, all with radius : a.

1. Emitting Ledge Model

To calculate the bubble diffusivity using this model, we assume local equilibrium at the facet and then write the probability that a given facet of radius a will have a ledge of critical size as $z \exp (-2 a \epsilon / k T)$ where $\epsilon$ is the edge energy and $z$ is the number of equivalent configurations. Then, following the Willertz and Shewmon treatment, but for emission rather than filling, the jump frequency of a given atom off the ledge is $4 D_{s} / \alpha_{s}^{2}$, where $\alpha_{s}$ is the average jump distance of a surface atom. The nucleation frequency $\Gamma$ then depends upon the probability that a critical nucleus exists multiplied by the rate at which it grows super-critical,

$$
\Gamma=\frac{2 a}{b} \frac{4 D_{s}}{\alpha_{s}^{2}} z \exp (-2 a \epsilon / k T),
$$

where $b$ is the atomic diameter. According to a surface diffusion study 24 at high temperatures, $\alpha_{s}$ could be of the order of 10 atomic distances. The random walk diffusivity in cubic crystals is given by $D=\Gamma \alpha^{2} / 6$. For bubble diffusion in isotropic solids, the average jump distance of a bubble when a layer has been removed from an emitting facet is $\alpha_{b}=b a^{2} / x^{2}$ where $b$ is the height of a step in the ledge. Then putting $8=a / r$ and $\lambda=\alpha_{s} / b$, we obtain for the bubble diffusivity, 


$$
D_{b}=\frac{4 z g^{5} D_{s} r}{3 \lambda^{2} b} \exp (-2 g \in r / k T)
$$

Assuming that a bubble has twenty-six facets of equal area, then $g=0.4$. If we take $z=\frac{\pi_{a}}{b}$, we obtain

$$
D_{b}=1.7 \times 10^{-2} \frac{D_{s}}{\lambda^{2}}\left(\frac{r}{b}\right)^{2} \exp (-0.8 \in r / k T) .
$$

2. Two-Pillbox Model

The total edge energy of all the facets on a bubble changes when atoms move from one facet to another. For simplicity we consider the exchange of atoms between just two facets. Furthermore we consider facets which are both originally atomically smooth. The ledge energy of the facets as atoms move from one facet to another creating two pillboxes will be

$$
\Delta G=2 \pi \epsilon\left(a_{1}+a_{2}\right),
$$

where $a_{1}$ and $a_{2}$ are the radil of the piliboxes in the emitting and filling facet, respectively. Since the total area of the atoms in the new layer formed around the edge of the filling facet is equal to the area of the pillbox on the filling facet, we can obtain

$$
\pi a_{1}^{2}=A-\pi a_{2}^{2}
$$

where $A$ is the area of a facet. The energy to form a critical nucleus $\Delta G^{*}$ can be obtained after combining the above equations and maximizing with respect to $a_{1}$,

$$
\Delta G^{*}=2 \epsilon(2 \pi A)^{\frac{1}{2}}
$$

Following a derivation similar to that for the emitting ledge model, we 
can obtain

$$
D_{b}=\frac{1}{6} z\left(\frac{2 \pi a^{*}}{b}\right) \frac{4 D_{s}}{\alpha_{s}^{2}} b^{2} g^{4} \exp \left(-\Delta G^{*} / k T\right),
$$

where $a^{*}=(A / 2 \pi)^{\frac{1}{2}}$ is the radius of a critical sized pillbox. Again, assuming that there are twenty-six facets in a bubble we have

$$
D_{b}=1.3 \times 10^{-3} \frac{D_{s}}{\lambda^{2}}\left(\frac{r}{b}\right)^{3} \exp (-3.55 \mathrm{er} / \mathrm{kT})
$$

\section{ACKNOWLEDGEMENTT}

The authors wish to thank S. Hruska and E. E. Gruber for helpful discussions and $M$. Oselka and the cyclotron staff at Argonne National Laboratory for their assistance in irradiation of the specimens. Financial support of this project by the U.S. Atomic Energy Commission under contract number AT (11-1) 1799 is acknowledged. 


\section{REFERENCES}

1. E. M. Baroody, J. Appl. Phys. 38 (1967) 4893

2. E. E. Gruber, J. Appl. Phys. 38 (1967) 243

3. G. W. Greenwood and M. V. Speight, J. Nucl. Mater. 10 (1963) 140

4. M. E. Gulder, J. Nucl. Mater. 23 (1967) 30

5. L. E. Willertz and P. G. Shewmon, Trans. Met. Soc. AIME $\underline{1}$ (1970) 2217

6. G. K. Walker, J. Nucl. Mater. 37 (1970) 171

7. J. R. Cost and D. I. Johnson, J. Nucl. Mater. 36 (1970) 230

8. J. P. Tartour and J. Washburn, Phil. Mag. 18 (1968) 1257

9. E. Ruedl, Irradiation Effects on Structural Alloys for Nuclear Reactor Applications, ASTM STP 484 (American Society for Testing and Materials 1970) pp $300-316$

10: J. Van Landuyt, R. Gevers, and S. Amelinckx, Phys. Stat. Sol. 10 (1965) 319

11. M. R. Rulhle, International Conference on Radiation-Induced Voids in Metals, Albany, 1971

12. S. Chandrasekhar, Rev. Mod. Phys. 15 (1943) 1

13. R. S. Nelson, D. J. Mazey and R. S. Barnes, Phil. Mag. Il (1965) 91

14. N. A. Gjostein, Adsorption Et Croissance Cristalline, Colloques International Du Centre National De La Recherche Scientifique, France, no. 52, 1965

15. N. A. Gjostein and F. N. Rhines, Acta Met. I (1959) 224

16. R. R. Hough, Scrip. Met. 4 (1970) 559

17. Handbook of Chemistry and Physics; ed., R. C. Weast, 49th Ed., (Chemical Rubber Co., Cleveland, 1968) p.D-107

18. R. S. Barnes, J. Nucl. Mater. 11 (1964) 135

19. P. W. Bridgman, Proc. Amer. Acad. Arts and Sci. 29 (1924) 173

20. W. G. Schneider and J.A.H. Duffie, J. Chem. Phys. 17 (1949) 751

21. J.W. Taylor, Acta Met. 4 (1956) 460 
22. H. Bethge, Molecular Processes in Solid Surfaces; ed., R. G. Gretz and R. I. Jaffee (McGraw-H11l, New York, 1969) 569

23. W. L. Winterbottom and N. A. Gjostein, Acta Met. 14 (1966) 1041

24. H. P. Bonzel and N. A. Gjostein, Molecular Processes in Solid Surfaces; ed., R. G. Gretz and R. I. Jaffee (McGraw-Hill, New York, 1969) 533 
TABLE I. Size distribution of helium bubbles obtained experimentally for various annealing times at $550^{\circ} \mathrm{C}$

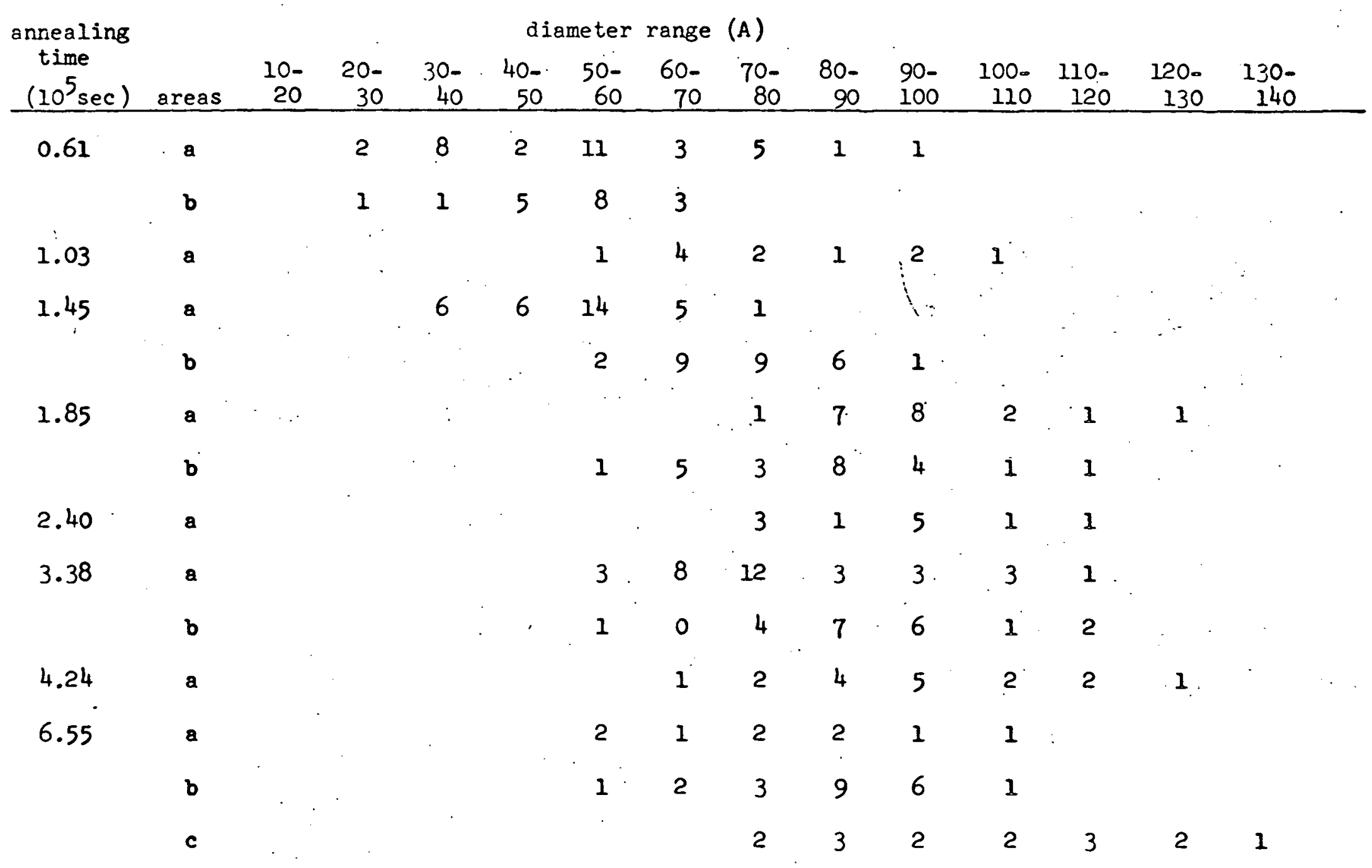


FIGURE CAPTIONS

Fig. 1. The difference in electrical resistivity of the irradiated and dummy specimen is plotted against annealing time at $550^{\circ} \mathrm{C}$.

Fig. 2. Electron micrograph of a specimen annealed at $550^{\circ} \mathrm{C}$ for $6.12 \times 10^{4}$ seconds. Many of the bubbles are clearly seen to be faceted.

Fig. 3. Electron micrograph of a specimen annealed at $550^{\circ} \mathrm{C}$ for $6.55 \times 10^{5}$ seconds. Contrast of bubbles changes across a bend extinction contour. The size distribution of bubbles is in a higher range than that shown in Fig. 2.

Fig. 4. Bubble size distribution obtained experimentally versus bubble radil and helium atoms in the bubbles. The result of Gruber's fit (curves) suggests a surface self-diffusion coefficient which is six to seven orders of magnitude too small, indicating that surface diffusion is not the rate-limiting step for bubble migration. The concentration of helium used in this calculation is $m=3 \times 10^{18}$ per $\mathrm{cm}^{3}$. The choice of the surface energy $\gamma=150$ ergs per $\mathrm{cm}^{2}$ is described in the discussion.

Fig. 5. Calculated bubble density is plotted against annealing time for surface diffusion-limited (for both a real and a Van der Waals gas) and facet nucleation-limited bubble motion. It is seen that the latter predicts a much slower coalescence rate than the former. The slope for the facet nuclation mechanism varies continuously and tends to decrease monotonically after long annealing times. The equation for the surface diffusion-limited mechanism is due to Gruber, and that for the facet nucleation-limited mechanism is developed in the Appendix. 
Fig. 6. The mean bubble radius obtained from computer calculation is plotted against the annealing time for the two different mechanisms shown in Fig. 5 .

Fig. 7. The mean bubble radius obtained from experiment is plotted against annealing time at $550^{\circ} \mathrm{C}$. Also the calculated results of coalescence for two different facet nucleation mechanisms are shown by the curves. The calculation was started from the first experimental data point, at $6.12 \times 10^{4} \mathrm{sec}$, so that the computer calculation oniy involves one conversion, thus minimizing the error from this source. The equations used are discussed in the Appendix.

Fig. 8. The number of helium atoms per bubble versus the bubble radius at $550^{\circ} \mathrm{C}$ for both a Van der Waals gas and the empiric relation obtained from Bridgman's high pressure data ${ }^{19}$. The results are shown for two different values for the surface energy of aluminum. 


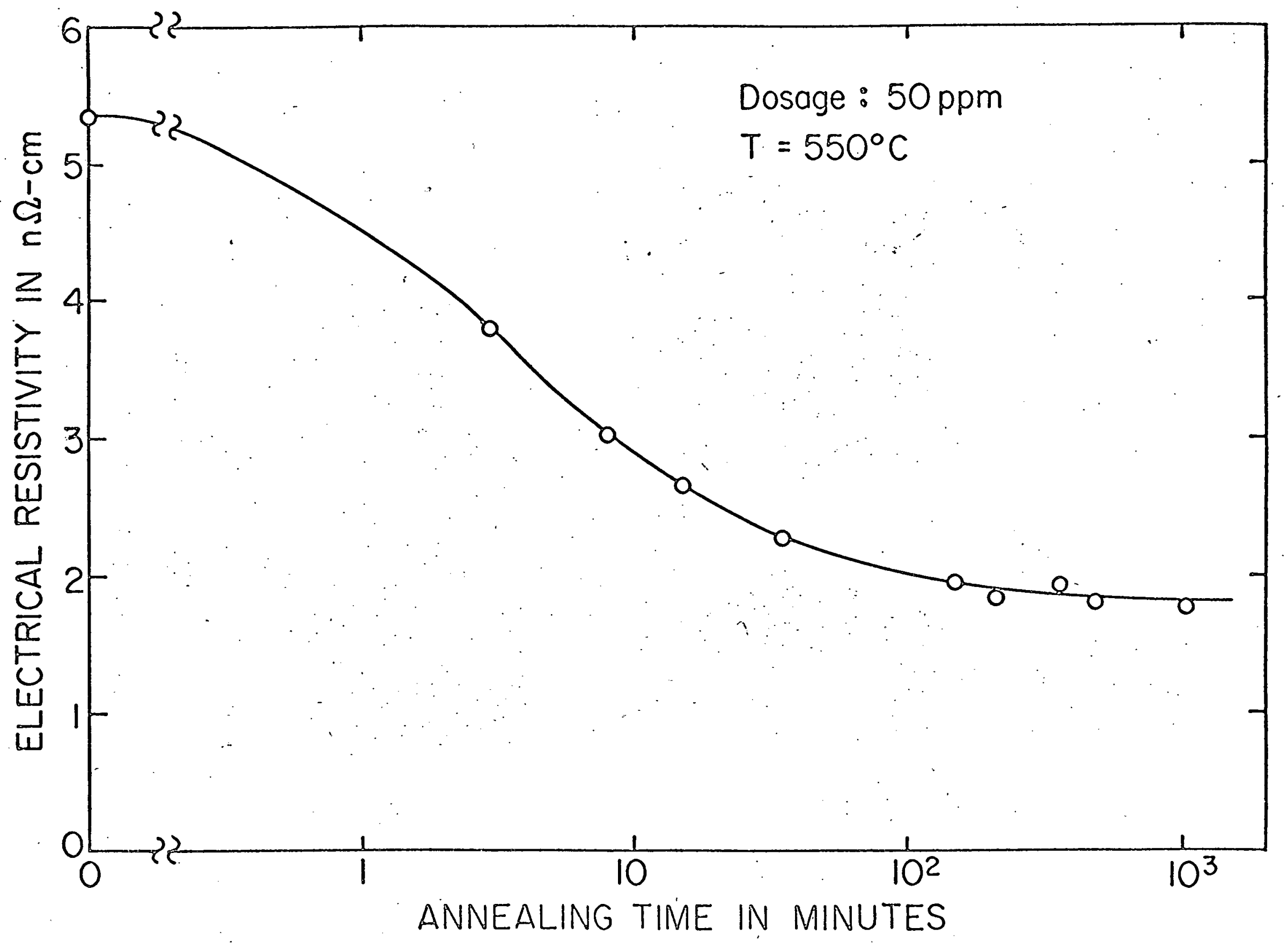

Figure 1 


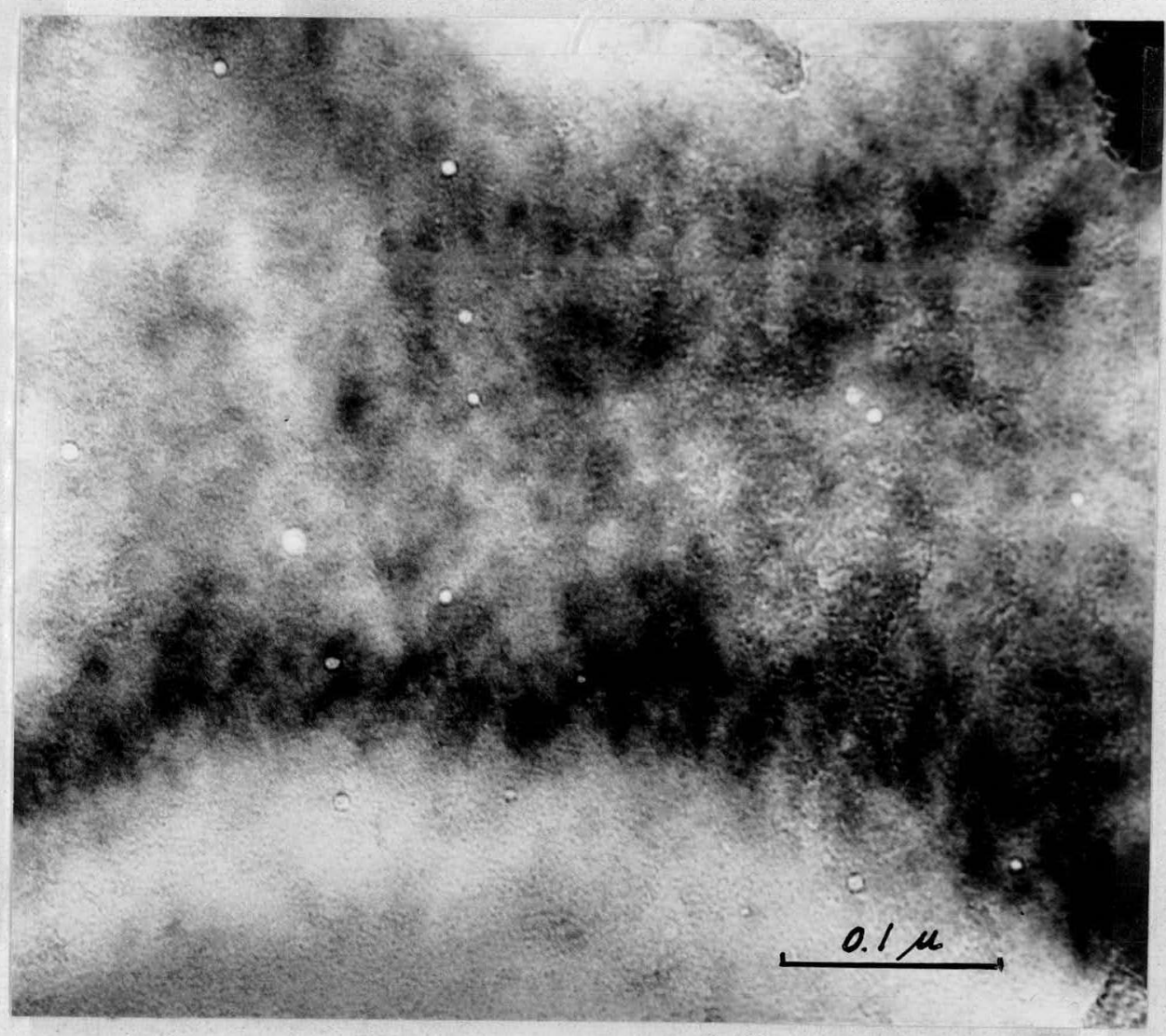

Figure 2

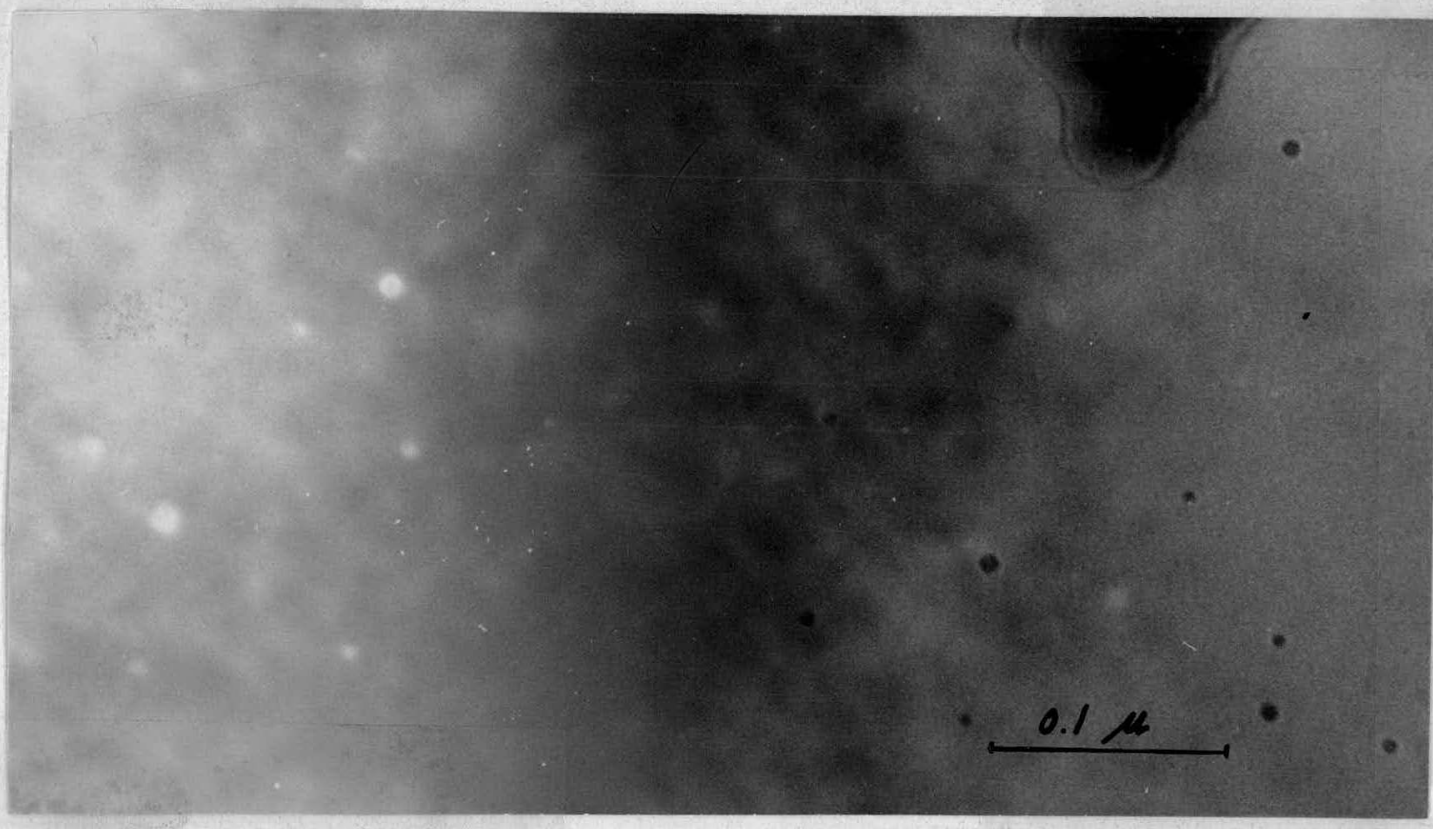

Figure 3 


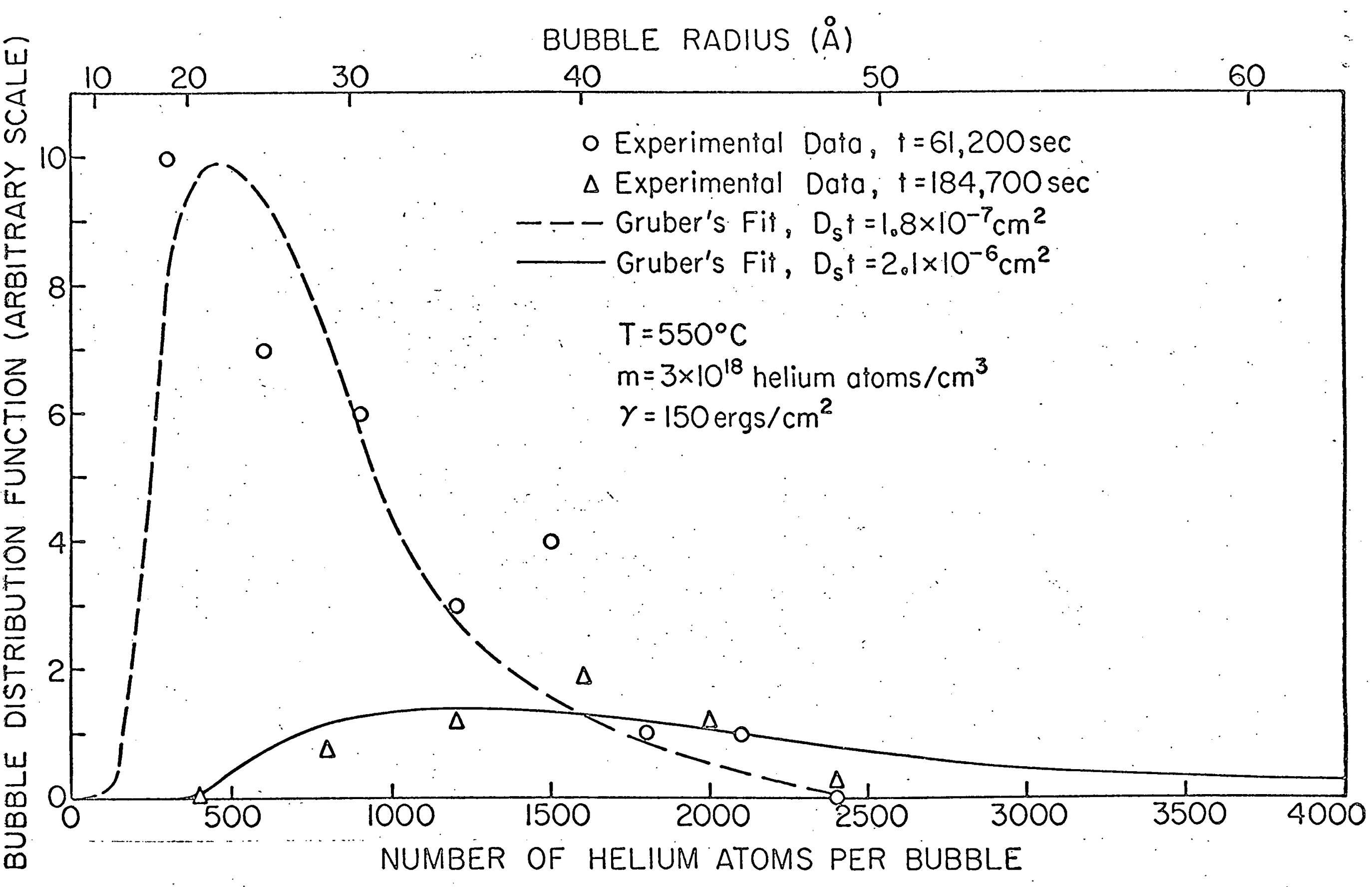

Figure 4 


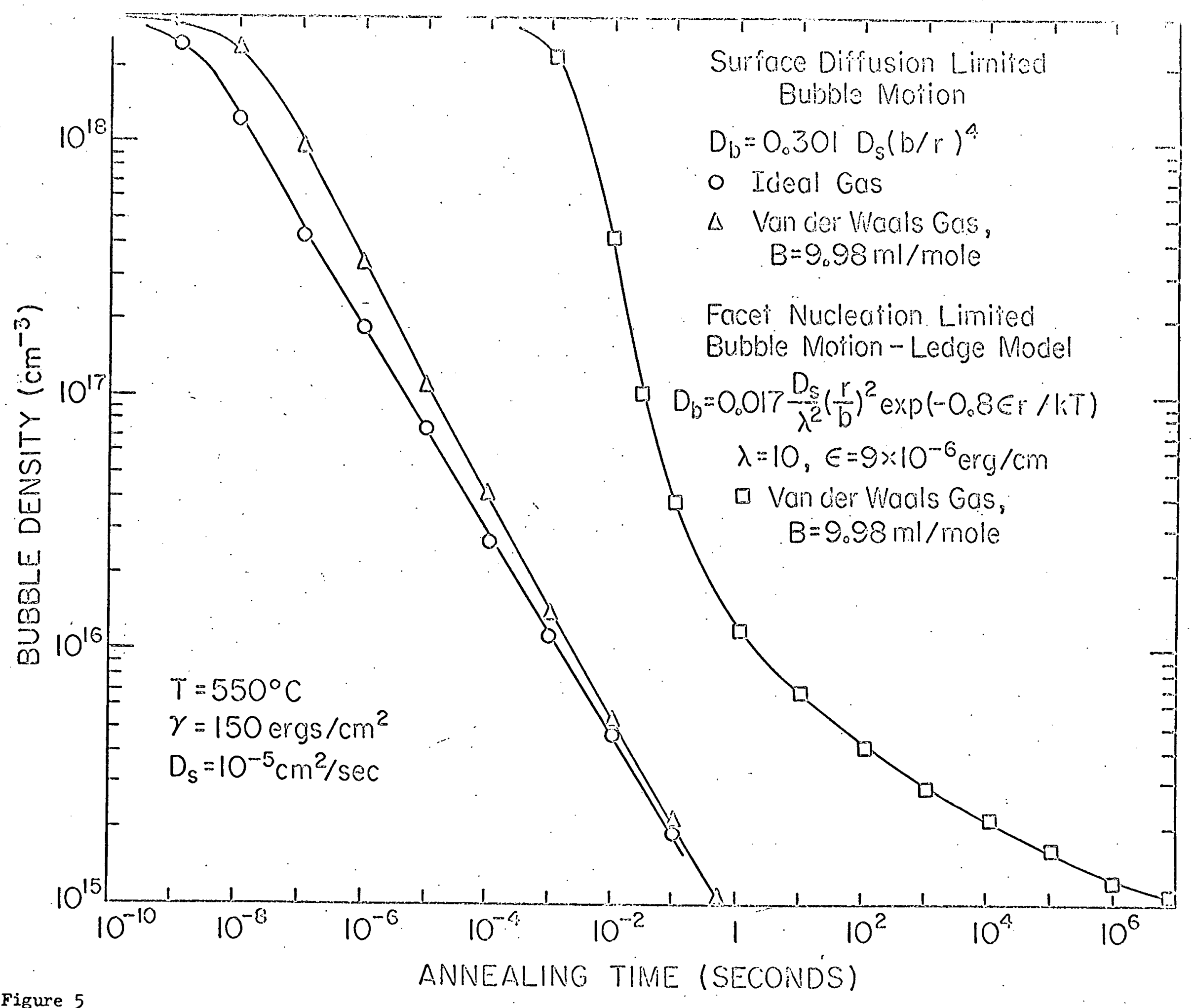




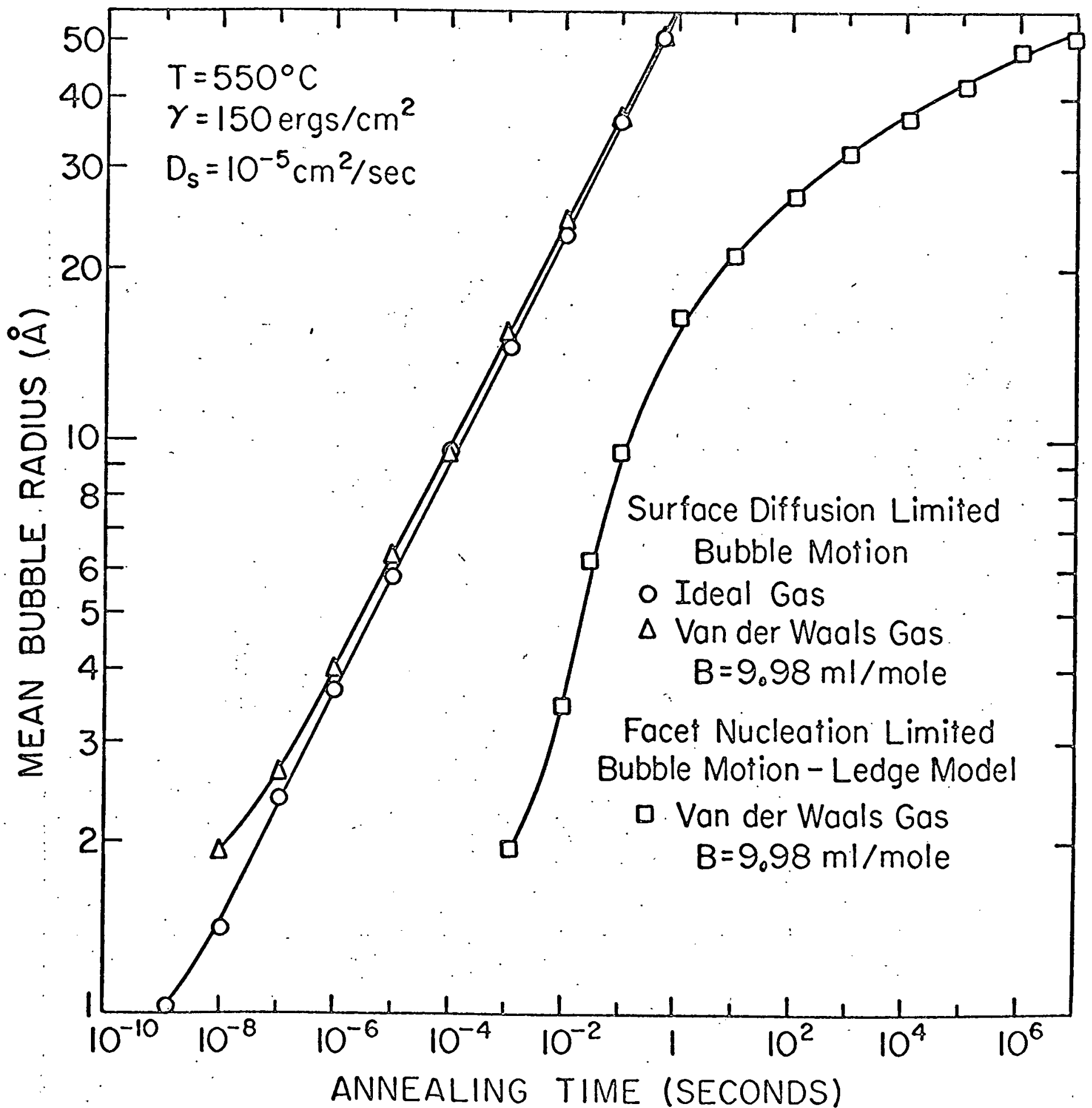

Figure 6 


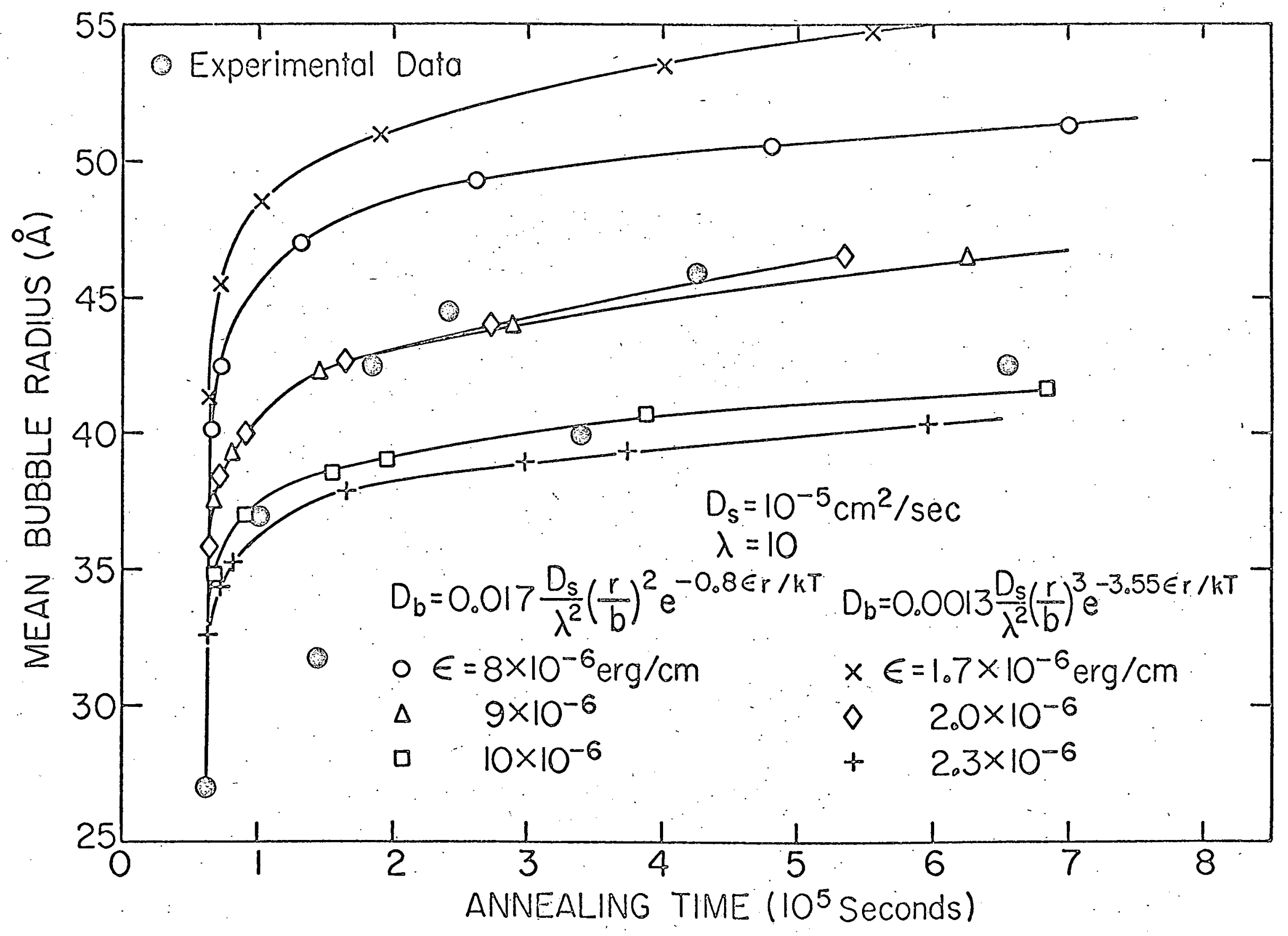




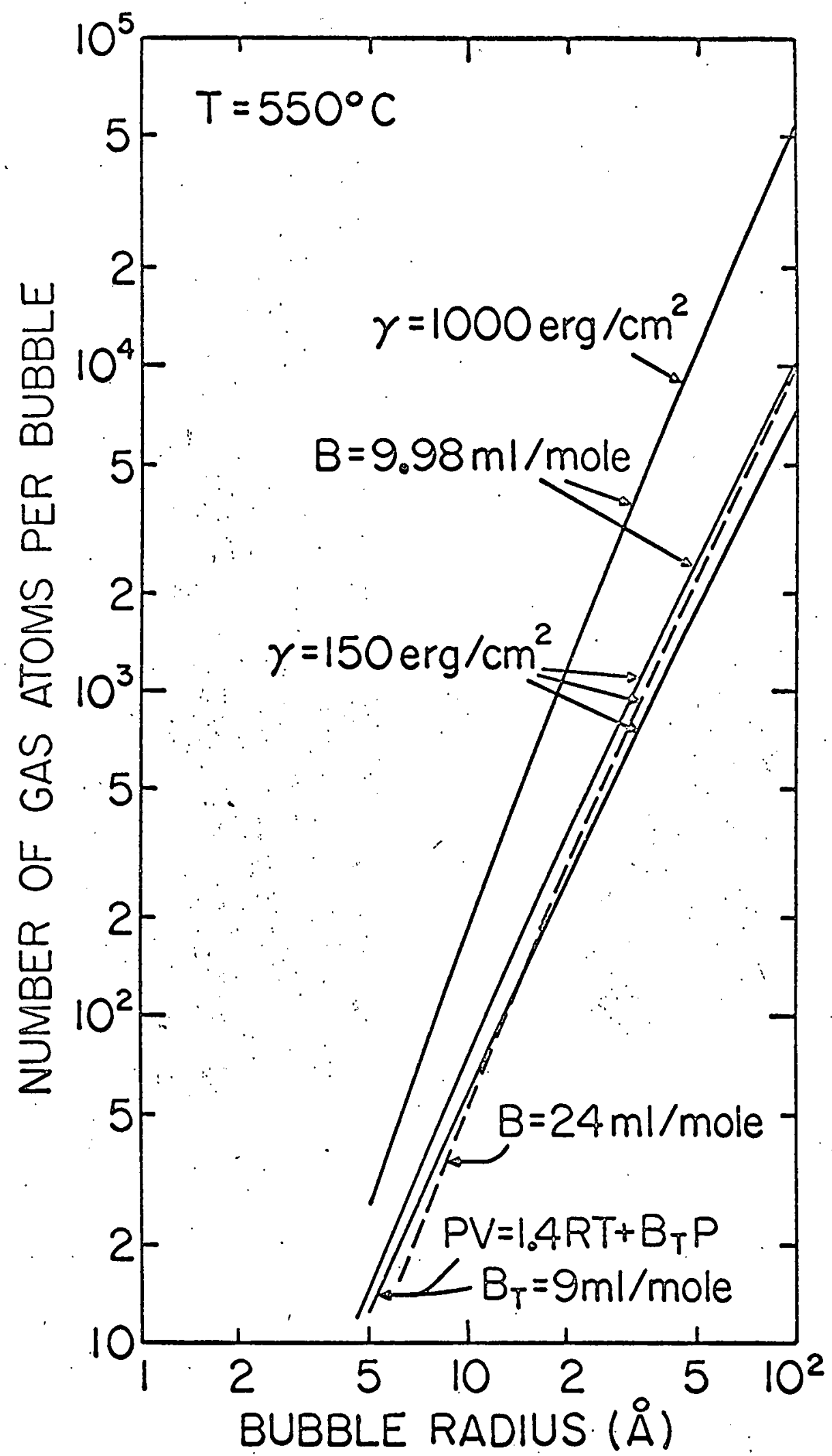

Figure 8 